\title{
Probing spin frustration in high-symmetry magnetic nanomolecules by inelastic neutron scattering
}

\author{
V. O. Garlea, ${ }^{1, *}$ S. E. Nagler, ${ }^{2}$ J. L. Zarestky, ${ }^{1}$ C. Stassis, ${ }^{1}$ D. Vaknin, ${ }^{1}$ P. Kögerler, ${ }^{1}$ D. F. McMorrow, ${ }^{3,4}$ C. Niedermayer, ${ }^{5}$ \\ D. A. Tennant, ${ }^{6}$ B. Lake, ${ }^{7,1}$ Y. Qiu, ${ }^{8}$ M. Exler, ${ }^{9}$ J. Schnack, ${ }^{9}$ and M. Luban ${ }^{1}$ \\ ${ }^{1}$ Ames Laboratory, Department of Physics and Astronomy, Iowa State University, Ames, Iowa 50011, USA \\ ${ }^{2}$ Oak Ridge National Laboratory, Oak Ridge, Tennessee 37831, USA \\ ${ }^{3}$ Ris $\phi$ National Laboratory, DK-4000, Roskilde, Denmark \\ ${ }^{4}$ Department of Physics and Astronomy, University College London, London, United Kingdom \\ ${ }^{5}$ Laboratory for Neutron Scattering ETHZ and PSI, CH-5232 Villigen PSI, Switzerland \\ ${ }^{6}$ School of Physics and Astronomy, University of St Andrews, St. Andrews, FIFE KY16 9SS, Scotland, United Kingdom \\ ${ }^{7}$ Clarendon Laboratory, University of Oxford, Parks Road, Oxford OX1 3PU, United Kingdom \\ ${ }^{8}$ NIST Center for Neutron Research, Gaithersburg, Maryland 20899, USA \\ and Department of Materials Science and Engineering, University of Maryland, College Park, Maryland 20742, USA \\ ${ }^{9}$ Universität Osnabrück, Fachbereich Physik, D-49069 Osnabrück, Germany \\ (Received 25 October 2005; revised manuscript received 14 December 2005; published 23 January 2006)
}

\begin{abstract}
Low temperature inelastic neutron scattering studies have been performed to characterize the low energy magnetic excitation spectrum of the magnetic nanomolecule $\left\{\mathrm{Mo}_{72} \mathrm{Fe}_{30}\right\}$. This unique highly symmetric cluster features spin frustration and is one of the largest discrete magnetic molecules studied to date by inelastic neutron scattering. The $30 \mathrm{~s}=5 / 2 \mathrm{Fe}^{\mathrm{III}}$ ions, embedded in a spherical polyoxomolybdate molecule, occupy the vertices of an icosidodecahedron and are coupled via nearest-neighbor antiferromagnetic interactions. The overall energy scale of the excitation and the gross features of the temperature dependence of the observed neutron scattering are explained by a quantum model of the frustrated spin cluster. However, no satisfactory theoretical explanation is yet available for the observed magnetic field dependence.
\end{abstract}

DOI: 10.1103/PhysRevB.73.024414 PACS number(s): 75.25.+z, 75.50.Ee, 75.75.+a, 78.70.Nx

\section{INTRODUCTION}

Magnetic molecules are confined assemblies of finite numbers of interacting spin centers, and are ideal systems for exploring fundamental issues in nanomagnetism, for example, quantum tunnelling, ${ }^{1}$ metastable magnetic phases, ${ }^{2}$ and spin frustration. ${ }^{3}$ Here we report inelastic neutron scattering results on the polyoxomolybdate $\left\{\mathrm{Mo}_{72} \mathrm{Fe}_{30}\right\}$. This molecule consists of 30 antiferromagnetically coupled $\mathrm{Fe}^{\mathrm{III}}$ ions $(s=5 / 2)$ that are linked between 12 pentagonal $\left\{\mathrm{Mo}_{6} \mathrm{O}_{21}\right\}$ fragments, resulting in an $I_{h}$-symmetric spherical cluster of $2.5 \mathrm{~nm}$ diameter in which the spin centers define the vertices of an icosidodecahedron. ${ }^{4,5}$ This polyhedron contains 20 corner-sharing $\mathrm{Fe}_{3}$ triangles, arranged around 12 pentagons [Fig. 1(a)]. Due to the icosahedral symmetry, a common antiferromagnetic superexchange interaction, $J S_{n} \cdot S_{m}$, links all nearest-neighbor spin centers resulting in spin frustration.

In its topology, $\left\{\mathrm{Mo}_{72} \mathrm{Fe}_{30}\right\}$ constitutes a molecular analogue of the Kagomé planar lattice, although in the latter corner-sharing triangles are grouped around hexagons. The spin frustration is easily visualized for the classical analogue of $\left\{\mathrm{Mo}_{72} \mathrm{Fe}_{30}\right\}$ where each $\mathrm{Fe}^{\mathrm{III}}$ is treated as a classical spin vector, i.e., free to assume any spatial orientation. The corresponding classical Heisenberg model results in a ground state configuration that can be described by three "sublattices" each consisting of 10 parallel spin vectors, with orientations defined by coplanar vectors offset by $120^{\circ}$ angles [Fig. 1(a)]. ${ }^{6}$ The application of an external magnetic field results in a uniform canting of the spins so that their projec- tions in the plane perpendicular to the magnetic field maintain the $120^{\circ}$ angle, until magnetic saturation occurs, at approximately 17.7 Tesla. $^{4}$

While the complete quantum Heisenberg spin Hamiltonian of $\left\{\mathrm{Mo}_{72} \mathrm{Fe}_{30}\right\}$, with a Hilbert space dimension of $6^{30}$, cannot be solved, a solvable approximate version has been derived for this system. ${ }^{7}$ An effective Hamiltonian has been
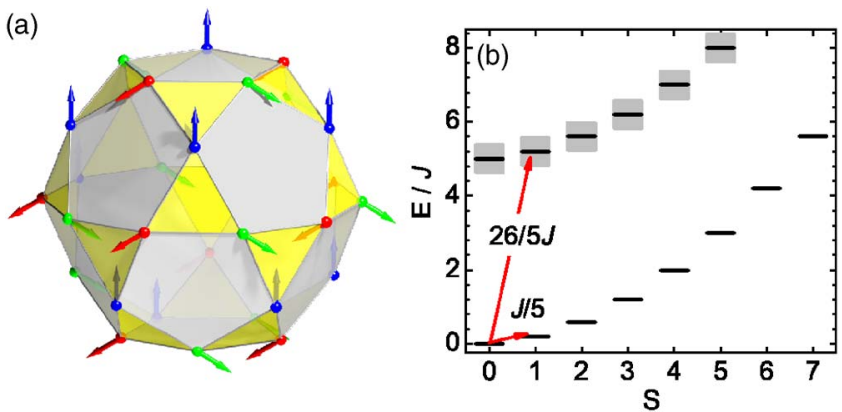

FIG. 1. (Color online) (a) The $30 \mathrm{Fe}^{\mathrm{III}}$ ions in $\left\{\mathrm{Mo}_{72} \mathrm{Fe}_{30}\right\}$ occupy the vertices of an icosidodecahedron. In a classical description at $0 \mathrm{~K}$ the 30 spins can be divided into three sublattices each of 10 parallel spins. There is a relative angle of $120^{\circ}$ for any pair of nearest-neighbor spin vectors. (b) Lowest energy levels calculated from a solvable quantum rotational band model (Ref. 7). Allowed transitions for the $S=0$ ground state to states with $S=1$ are indicated. The energy levels of the first excited rotational band are shown shaded (schematic only) to reflect the fact that the high degeneracy of these levels is partially lifted for the realistic nearestneighbor Heisenberg model of $\left\{\mathrm{Mo}_{72} \mathrm{Fe}_{30}\right\}$. 
constructed so that its classical version correctly reproduces the three-sublattice ground state spin configuration of the classical $\left\{\mathrm{Mo}_{72} \mathrm{Fe}_{30}\right\}$ spin array. ${ }^{7}$ The quantum version of this effective Hamiltonian is easily solved and provides a magnetic excitation spectrum in which the individual levels are characterized by the total spin quantum number $S$ and the total spin quantum numbers, $S_{A}, S_{B}$, and $S_{C}$, of the three sublattices. The system has an $S=0$ ground state while the lower section of the excitation spectrum features a set of parallel "rotational bands" [Fig. 1(b)], where the discrete energies are proportional to $S(S+1)$ and the energy gap separating the two lowest rotational bands is $5 \mathrm{~J}$. The experimental determination of the magnetic excitation spectrum is pivotal for comparison with theoretical models, and the inelastic neutron scattering technique has been most effective in this regard. ${ }^{8-13}$ Our study provides therefore the opportunity to test to what extent the gross features of the actual excitation spectrum of $\left\{\mathrm{Mo}_{72} \mathrm{Fe}_{30}\right\}$ are explained by the effective Hamiltonian based on rotational bands of excitations within a frustrated three-sublattice spin structure.

\section{EXPERIMENTAL RESULTS}

The inelastic neutron scattering experiments reported here were performed on deuterated $\left\{\mathrm{Mo}_{72} \mathrm{Fe}_{30}\right\}$ samples to minimize the attenuation and incoherent scattering from the hydrogen atoms. Characterization of the deuterated samples by magnetic susceptibility measurements, infrared and Raman spectroscopy, and single crystal and powder x-ray diffraction confirmed that their properties were consistent with those of nondeuterated samples studied earlier. ${ }^{4}$ The neutron measurements used approximately $10 \mathrm{~g}$ of polycrystalline samples sealed in copper holders under He atmosphere. The inelastic neutron spectra were collected at low temperatures using three different spectrometers: RITA2 at PSI, ${ }^{14}$ DCS at NCNR, ${ }^{15}$ and OSIRIS at ISIS. ${ }^{16}$ The data presented here were obtained at OSIRIS using a fixed final neutron energy $E_{f}=1.845 \mathrm{meV}$. Figure 2 shows intensities (integrated over the range $Q=0.9$ to $1.8 \AA^{-1}$ ) vs neutron energy loss at various temperatures. The upper panel [Fig. 2(a)] shows a semi$\log$ plot of the spectrum at the base temperature, nominally $T=65 \mathrm{mK}$. The large elastic peak has a FWHM of 0.021 (1) $\mathrm{meV}$ consistent with the instrumental energy resolution. A much broader peak is visible as a shoulder in the plot. The lower panel [Fig. 2(b)] shows the scattering on a linear scale at four different temperatures, ranging up to $6.5 \mathrm{~K}$. At base temperature a clear peak is evident near an energy transfer of $0.6 \mathrm{meV}$. As the temperature is raised this peak weakens, and there is a notable increase in the intensity of the scattering at low energies. This behavior of the intensity is expected if the peak at $\sim 0.6 \mathrm{meV}$ is due to a magnetic transition from the ground state. The temperature-enhanced low energy signal could in principle arise from lattice vibrations or from magnetic scattering, and differentiating between these possibilities requires careful analysis. ${ }^{17}$ To extract quantitative information, the base temperature scattering was fit to a simple model, assuming that the instrumental resolution at the elastic position can be described by the sum of two co-centered peaks: a dominant Gaussian

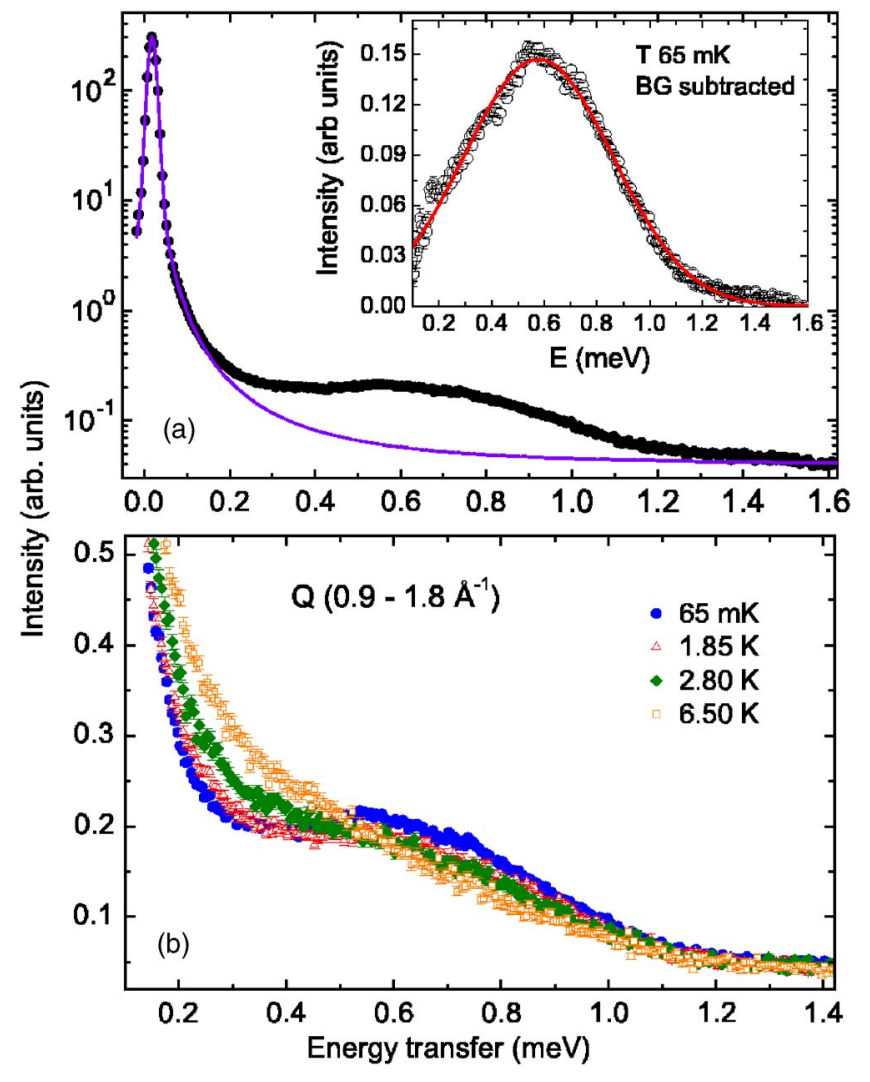

FIG. 2. (Color online) (a) Inelastic neutron scattering spectrum at a nominal temperature of $65 \mathrm{mK}$. The intensity axis is on a logarithmic scale. The solid line shows a best estimate of the nonmagnetic background (see text). Inset: the background subtracted scattering. (b) Raw data for several different temperatures plotted on a linear scale.

and a Lorentzian with the latter accounting for the extended tails. The nonmagnetic background scattering is taken as the sum of an elastic term proportional to the resolution function plus an energy independent constant. The magnetic scattering is represented by a single Gaussian. The solid curve in Fig. 2(a) illustrates the background, and the inset shows the background subtracted signal fit to a single Gaussian with peak position $0.56(1) \mathrm{meV}$ and FWHM 0.66(1) meV. As seen in the inset to Fig. 2(a) the overall distribution can be modeled by a single peak, however the data shows additional one or more shoulders on the low energy side. The observed energy widths of the shoulders and the overall distribution are intrinsic since the resolution is roughly FWHM $0.02 \mathrm{meV}$.

To gain more insight into the nature of the magnetic excitations, we examined their temperature dependence. The background subtracted scattering for several temperatures up to $6.5 \mathrm{~K}$ is shown in Fig. 3. A thorough analysis of the data indicates that the contribution of single and multiple phonon scattering from solids or fluids is insignificant over the wave vector and temperature range of the data considered here, ${ }^{17,18}$ substantiating the scattering to be magnetic. Furthermore, the strong effect of external magnetic field on the excitations, discussed below, is clear evidence that the backgroundsubtracted inelastic scattering (Fig. 3) is dominated by mag- 


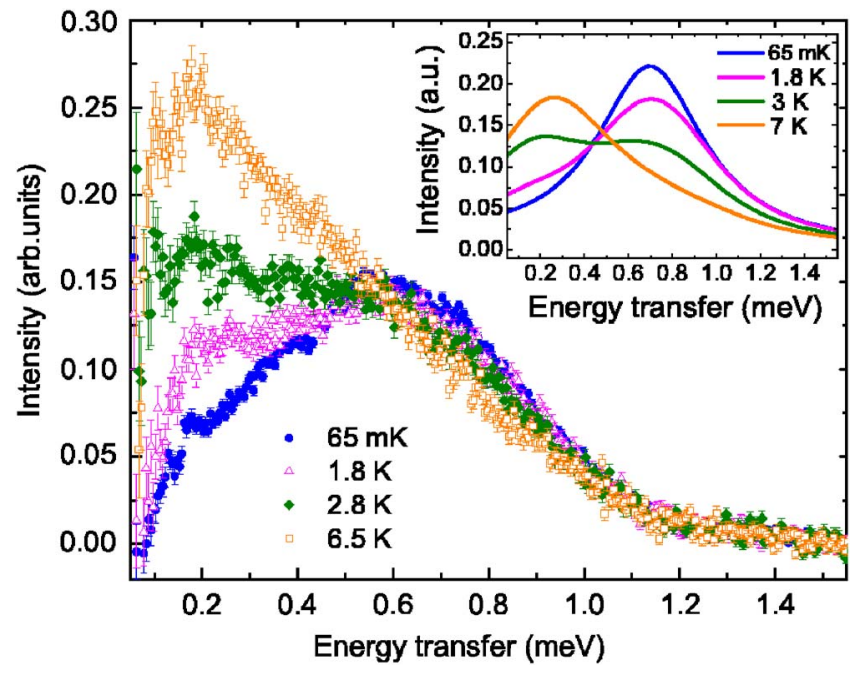

FIG. 3. (Color online) Temperature dependence of the inelastic neutron scattering, plotted with the nonmagnetic background subtracted. Inset, theoretical scattering for several temperatures, calculated using the quantum rotational band model with simplifying assumptions (see text and Ref. 20).

netic transitions in $\left\{\mathrm{Mo}_{72} \mathrm{Fe}_{30}\right\}$. With the uncertainties in instrumental resolution there may be some amount of magnetic scattering included as background at low energy transfers, possibly contributing to an over-subtraction at energy transfers below $0.2 \mathrm{meV}$. The magnetic nature of the observed spectrum was confirmed by studying the effect of an externally applied magnetic field on $\left\{\mathrm{Mo}_{72} \mathrm{Fe}_{30}\right\}$. Figure 4 shows the background subtracted energy scans for 0 and 7 Tesla (extracted similarly to the scans shown in Fig. 3). At 7 Tesla, the main excitation is lower in intensity and two side-bands, at lower and higher energies, are observed. Our analysis shows the position of the new bands is roughly linear in

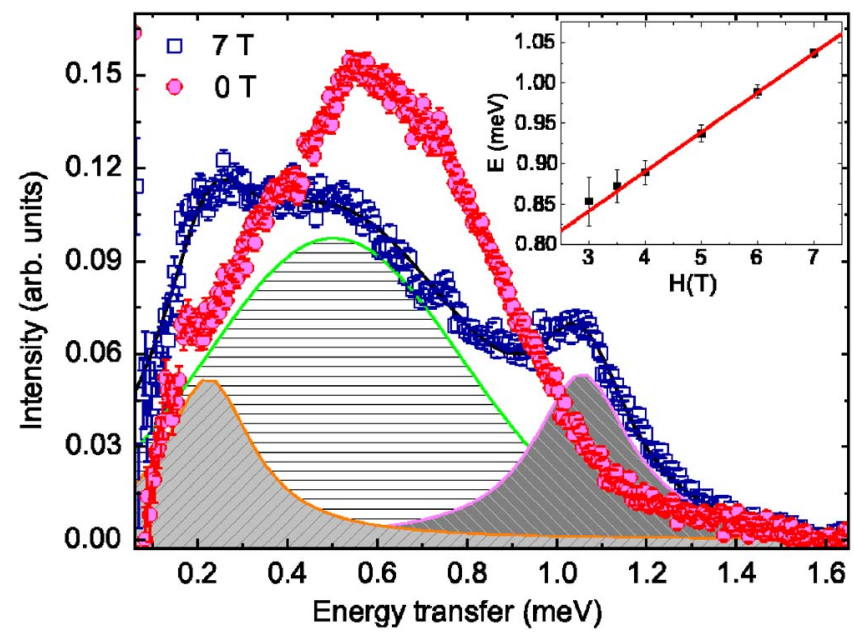

FIG. 4. (Color online) Intensities versus neutron energy-loss showing the effect of a 7 Tesla magnetic field on the main excitation. In an applied magnetic field, the intensity of the main excitation peak decreases and two side-bands at lower and higher energies are observed. The solid line describes the convolution of the three shaded bands for the 7 Tesla data. Inset, linear field dependence of the high energy side-band. magnetic field strength. ${ }^{17}$ The position of the central band shifts slightly with the applied field; the value at 7 Tesla is estimated as $0.50(1) \mathrm{meV}$. Following the detailed field dependence of the low energy side-band quantitatively is difficult because of the large background at low energy transfers. The high energy side-band can be observed more clearly, and the peak position as a function of field is plotted in the inset to Fig. 4. For small fields the side peaks cannot be resolved, but over the visible range the slope of the upper peak is 0.049(1) meV Tesla ${ }^{-1}$.

\section{DISCUSSION}

We now consider these results in light of the existing theoretical descriptions for the excitations in $\left\{\mathrm{Mo}_{72} \mathrm{Fe}_{30}\right\}$. The exactly solvable model discussed previously ${ }^{7}$ is obtained by defining the effective Hamiltonian operator, in the absence of an external magnetic field and the absence of single-spin anisotropy terms as appropriate for $\mathrm{Fe}^{\mathrm{IIII}}$ ions in an octohedral ligand field, as $\tilde{\mathbf{H}}=(J / 5)\left(\widetilde{\mathbf{S}}_{A} \cdot \widetilde{\mathbf{S}}_{B}+\widetilde{\mathbf{S}}_{B} \cdot \widetilde{\mathbf{S}}_{C}+\widetilde{\mathbf{S}}_{C} \cdot \widetilde{\mathbf{S}}_{A}\right)+195 J .^{7}$ The resulting spectrum, with ground state energy zero, has a hierarchy of discrete energy levels for each value of the total spin quantum number $S$ given by $E(S)=(J / 10)[S(S+1)$ $\left.-S_{A}\left(S_{A}+1\right)-S_{B}\left(S_{B}+1\right)-S_{C}\left(S_{C}+1\right)\right]+195 J$, where the spin quantum numbers span the range $0 \leqslant S \leqslant 75$ and $0 \leqslant S_{A, B, C}$ $\leqslant 25$. In particular, the energy levels of the lowest rotational band, for which $S_{A}=S_{B}=S_{C}=25$, are given by $E_{1}(S)$ $=(J / 10) S(S+1)$; for the first excited rotational band, characterized by $S_{A}=24, S_{B}=S_{C}=25$ and the permutations thereof, $E_{2}(S)=5 J+E_{1}(S)$. The corresponding degeneracies, for $S$ $\leqslant 24$, are $D_{1}=d_{1}(S) \cdot(2 S+1)$, for the lowest, and $D_{2}$ $=d_{2}(S) \cdot(2 S+1)$ for the first excited rotational band. Here $d_{1}(S)=2 S+1$ and $d_{2}(S)=27(2 S+1)$ are the $S$-degeneracy factors for these bands. These results follow upon using the standard rules for coupling angular momenta. At $T=0$ neutron scattering is dominated by transitions from the $S=0$ ground state to states with $S=1$. This implies a gapped mode with energy $J / 5$ for the transition within the lowest rotational band, and higher energy mode with an energy of $(26 / 5) J$ for the interband transition shown in Fig. 1(b). Susceptibility measurements ${ }^{4}$ provide a rough value of $J \approx 0.134 \mathrm{meV}$, implying that the intraband mode should appear near $27 \mu \mathrm{eV}$. The large quasielastic background precludes an observation of such a low energy mode in the present experiment. On the other hand, the predicted interband transition energy is $26 / 5 \mathrm{~J} \approx 0.69 \mathrm{meV}$, in rough agreement with the overall energy scale of the observed neutron excitations at $\sim 0.6 \mathrm{meV}$ as seen in Fig. 2. As mentioned previously, the lowest temperature scattering seen in the inset to Fig. 2(a) has an intrinsic width and structure that cannot be explained by the exactly solvable three-sublattice model. Recently boson operator methods have been applied to the nearest neighbor Heisenberg Hamiltonian, appropriate for $\left\{\mathrm{Mo}_{72} \mathrm{Fe}_{30}\right\}$, to obtain approximate solutions for the $S=1$ excitations. ${ }^{19}$ This calculation predicts that several modes should be visible in the region of interest. Interestingly, the predicted energy levels are in rough agreement with the overall linewidth and locations of the additional spectral structure observed in the 
experiment, although the observed structure is much broader than the resolution limited line shapes expected from this calculation. The detailed line shape of the base temperature scattering may therefore reflect the richer structure of the energy spectrum of the nearest-neighbor Heisenberg model, as compared to that of the three-sublattice rotational band model. It may also reflect the effects of weak anisotropy. Even at base temperature the experimental line shapes may be affected by thermal broadening. A full understanding of the line shape requires further theoretical work.

Notwithstanding the details of the base temperature line shape, one can still consider the overall temperature dependence of the scattering as shown in Fig.3 within the context of the rotational band model. As the temperature increases above $T \geqslant J / 5 \approx 0.3 \mathrm{~K}$, energy levels within the lowest band become more populated, giving rise to the broadening and shifting of the main excitation towards $E=0$. A calculation was carried out starting from standard formulas for magnetic neutron scattering, ${ }^{18}$ using a single common nonzero matrix element for all allowed transitions. ${ }^{20}$ Thermal occupation of levels was accounted for by a Boltzmann factor, and Dirac delta function factors associated with allowed transitions were replaced by a Lorentzian of $0.3 \mathrm{meV}$ width. Calculated curves using $J=0.134 \mathrm{meV}$ are in qualitative agreement with the measured data as shown in the inset to Fig. 3. As shown, for successively higher temperatures the calculated peak broadens and shifts to lower energies. Near $7 \mathrm{~K}$ the peak in the intensity occurs at around $0.2 \mathrm{meV}$, similar to that seen experimentally. The rotational band model thus can provide a reasonable explanation of the temperature behavior of the neutron spectrum for $\left\{\mathrm{Mo}_{72} \mathrm{Fe}_{30}\right\}$. The replacement of the Dirac delta function factors by a broad Lorentzian so as to secure qualitative agreement with the base temperature scattering is supported by the fact that application of the correct Heisenberg model of $\left\{\mathrm{Mo}_{72} \mathrm{Fe}_{30}\right\}$ partially lifts the large degeneracies of the energies of the approximate three-sublattice rotational band model. Calculation of the dimensionalities of the irreducible representations of the icosahedral group in the case of the icosidodecahedron leads to the result that the maximum value of the $S$-degeneracy factor $d(S)$ for any individual level in the nearest-neighbor model is 5, or possibly higher if there are additional accidental degeneracies. Thus, for example, an energy level of the first excited rotational band, for which $d_{2}(S)=27(2 S+1)$, would correspond to multiple, distinct energies in the full model each with a reduced value of $d(S)$.

The measurements in nonzero fields should, in principle, provide a stringent test of the existing theoretical models for $\left\{\mathrm{Mo}_{72} \mathrm{Fe}_{30}\right\}$. A straightforward extension of the rotational band model to nonzero fields predicts that at low temperatures the excitation spectrum should have three main peaks. One of these is unshifted from the zero field peak, and gradually loses intensity as the field is increased. The other two have energies depending linearly on the field strength, but in contrast to the results illustrated in Fig. 4, both peaks occur at energies greater than the zero field peak, with slopes different from that observed in the experiment. At present the reasons for these discrepancies are unknown. It should be remarked that the precise degeneracies of the peaks affect the experimental response in a field quite strongly, so some deviations from the predictions of the rotational band model are expected.

\section{SUMMARY}

In summary, inelastic neutron scattering data at low temperatures and under applied magnetic field have provided the first determination of the magnetic excitations of the $s=5 / 2$ icosidodecahedron Keplerate $\left\{\mathrm{Mo}_{72} \mathrm{Fe}_{30}\right\}$. We found that the solvable three-sublattice model $^{7}$ for describing the frustrated spin system accounts well for the overall energy scale and qualitative temperature dependence of our experimental observations. The principal mode observed can be understood as arising from transitions between the two lowest rotational bands. The results obtained in various magnetic fields confirmed the magnetic origin of the spectrum but challenge the existing theoretical models. An improved quantitative treatment of the detailed line shape at base temperature and an understanding of the behavior in a magnetic field may require a more sophisticated theoretical approach. More detailed presentations of the experimental and thoretical aspects of this investigation are in preparation. ${ }^{17,20}$

\section{ACKNOWLEDGMENTS}

The authors thank R. J. McQueeney and B. Normand for helpful discussions. The authors also thank H.-J. Schmidt for providing us with the maximum $S$-degeneracy factor that can occur for the nearest-neighbor Heisenberg model of spins on an icosidodecahedron. Work at ORNL is supported by the U.S. DOE under Contract No. DE-AC05-00OR22725 with UT-Battelle, LLC and work at Ames Laboratory was supported under Contract No. W-7405-Eng-82. The work at NIST was supported in part by the NSF under Contract No. DMR-0086210. The work at Universität Osnabrück was supported by DFG Grand No. SCHN-615/5-2. The OSIRIS measurements (D.A.T., B.L.) were supported by UK EPSRC Grant No. GR/N35038/01.

\footnotetext{
*Electronic address: garleao@ornl.gov

${ }^{1}$ D. Gatteschi, Magnetic Molecules, NATO Science Series, II: Mathematics, Physics and Chemistry, Vol. 139 (Organic Conductors, Superconductors and Magnets: From Synthesis to Molecular Electronics, edited by L. Ouahab and E. Yagubskii) (Klu-
}

wer Academic, Dordrecht, 2004), pp. 179-196.

${ }^{2}$ C. Schröder, H. Nojiri, J. Schnack, P. Hage, M. Luban, and P. Kögerler, Phys. Rev. Lett. 94, 017205 (2005).

${ }^{3}$ See, e.g., J. Schnack, Quantum Magnetism, Lect. Notes Phys. Vol. 645 (Springer, Berlin, 2004). 
${ }^{4}$ A. Müller, M. Luban, C. Schröder, R. Modler, P. Kögerler, M. Axenovich, J. Schnack, P. C. Canfield, S. Bud'ko, and N. Harrison, ChemPhysChem 2, 517 (2001).

${ }^{5}$ Although the geometries of the individual $\left\{\mathrm{Mo}_{72} \mathrm{Fe}_{30}\right\}$ clusters do not exactly confirm the $I_{h}$ point group, the $\mathrm{Fe}_{30}$ spin frameworks are very close to the $I_{h}$ ideal, whereby a standard deviation of $0.04525 \AA(0.70 \%)$ from the mean Fe-Fe contact distance of $6.4493 \AA$ is observed. Given the weak distance dependency of the exchange coupling for -O-Mo-O-pathways in magnetically functionalized polyoxomolybdates, such variations are insignificant in the context of this paper.

${ }^{6}$ M. Axenovich and M. Luban, Phys. Rev. B 63, 100407(R) (2001).

${ }^{7}$ J. Schnack, M. Luban, and R. Modler, Europhys. Lett. 56, 863 (2001).

${ }^{8}$ M. Hennion, L. Pardi, I. Mirebeau, E. Suard, R. Sessoli, and A. Caneschi, Phys. Rev. B 56, 8819 (1997).

${ }^{9}$ Y. Zhong, M. P. Sarachik, J. R. Friedman, R. A. Robinson, T. M. Kelley, H. Nakotte, A. C. Christianson, F. Trouw, S. M.J. Aubin, and D. N. Hendrickson, J. Appl. Phys. 85, 5636 (1999).

${ }^{10}$ G. Chaboussant, A. Sieber, S. Ochsenbein, H. -U. Güdel, M. Murrie, A. Honecker, N. Fukushima, and B. Normand, Phys.
Rev. B 70, 104422 (2004).

${ }^{11}$ R. Caciuffo, G. Amoretti, A. Murani, R. Sessoli, A. Caneschi, and D. Gatteschi, Phys. Rev. Lett. 81, 4744 (1998).

${ }^{12}$ S. Carretta, J. van Slageren, T. Guidi, E. Liviotti, C. Mondelli, D. Rovai, A. Cornia, A. L. Dearden, F. Carsughi, M. Affronte, C. D. Frost, R. E. P. Winpenny, D. Gatteschi, G. Amoretti, and R. Caciuffo, Phys. Rev. B 67, 094405 (2003).

${ }^{13}$ O. Waldmann, T. Guidi, S. Carretta, C. Mondelli, and A. L. Dearden, Phys. Rev. Lett. 91, 237202 (2003).

${ }^{14}$ Details of the RITA2 spectrometer, PSI are found at http:// rita2.psi.ch

${ }^{15}$ See, e.g., J. R.D. Copley and J. C. Cook, Chem. Phys. 292, 477 (2003).

${ }^{16}$ Details of OSIRIS are found at http://www.isis.rl.ac.uk/ molecularspectroscopy/osiris/

${ }^{17}$ V. O. Garlea et al. (unpublished).

${ }^{18}$ See, e.g., V. F. Turchin, Slow Neutrons (Sivan Press, Jerusalem, 1995); S. W. Lovesey, Theory of Neutron Scattering from Condensed Matter (Clarendon, New York, 1984).

${ }^{19}$ O. Cépas and T. Ziman, Prog. Theor. Phys. Suppl. 159, 280 (2005).

${ }^{20}$ M. Exler, J. Schnack, and M. Luban (unpublished). 